\title{
BRANCHED CYCLIC COVERS OF SIMPLE KNOTS ${ }^{1}$
}

\author{
PAUL STRICKLAND
}

\begin{abstract}
The Blanchfield pairing of simple $(2 q-1)$-knots, $q \geqslant 2$, is used to give an algebraic characterization of those knots which may arise as $m$-fold branched cyclic covers of simple knots.
\end{abstract}

0. Introduction. In this paper we use the classification of odd-dimensional simple knots in terms of their Blanchfield pairing in [K and $\mathbf{T}]$ to give an algebraic characterization of those knots which can arise as branched cyclic covers of other knots.

We work in the piecewise linear category throughout, where all embeddings and isotopies are taken to be locally flat. An $n$-knot is an embedding $S^{n} \hookrightarrow S^{n+2}$, where the spheres are oriented; two such knots $k, l$ are equivalent if there is an isomorphism of the pairs $\left(S^{n+2}, k S^{n}\right),\left(S^{n+2}, l S^{n}\right)$.

I would like to express my thanks to Cherry Kearton for suggesting this line of research, and for pointing out a direct proof for Theorem 1.

1. Suppose we have an odd-dimensional simple knot $k=\left(S^{2 q+1}, S^{2 q-1}\right), q \geqslant 2$, with Alexander module $A_{t}$ and Blanchfield pairing $\langle,\rangle_{t}$, the $t$ subscript denoting a generator for the group of covering translations of $\tilde{X}$, the infinite cyclic cover of the exterior $X$ of the knot. We wish to determine conditions on the module and pairing which will allow $k$ to arise as the $m$-fold branched cyclic cover of another knot $l$. If $k$ does so arise, then $\tilde{X}$ will also be the infinite cyclic cover of $l$; but the group of covering translations will now be generated by a homeomorphism $u$ such that $u^{m}=t$. This homeomorphism will allow us to consider $A_{t}$ as a $\mathbf{Z}\left[u, u^{-1}\right]$-module $A_{u}$, which will be the Alexander module of $l$. It will also enable us to write down the Blanchfield pairing $\langle,\rangle_{u}$ of $l$ as follows.

Take two elements $a, b$ of $A_{t}=H_{q}(\tilde{X}) . A_{t}$ is a $\Lambda$-torsion module $\left(\Lambda=\mathbf{Z}\left[t, t^{-1}\right]\right)$, so there exists a nonzero $\pi(t) \in \Lambda$ such that $\pi a=0$. Choose a triangulation of $\tilde{X}$ induced by a triangulation of the exterior of $l$, and let $C_{q}$ be the group of $q$-chains, $\tilde{C}_{q+1}$ the group of $(q+1)$-chains in the dual triangulation. As $\pi a=0$, we may choose an element $\alpha$ of $\tilde{C}_{q+1}$ whose boundary represents $\pi a$; we also choose $\beta \in C_{q}$ representing $b$. Then we define $[\mathbf{B}, \mathbf{K}]$

$$
\langle a, b\rangle_{t}=\left[\left(\sum_{i=-\infty}^{\infty} I\left(\alpha, t^{i} \beta\right) t^{i}\right) / \pi(t)\right] \in\left(Q(t) / \mathbf{Z}\left[t, t^{-1}\right]\right),
$$

Received by the editors January 31, 1983.

1980 Mathematics Subject Classification. Primary 57Q45.

'Research supported by an SERC grant at Durham University. 
and

$$
\langle a, b\rangle_{u}=\left[\left(\sum_{i=-\infty}^{\infty} I\left(\alpha, u^{i} \beta\right) u^{i}\right) / \pi\left(u^{m}\right)\right] \in\left(Q(u) / \mathbf{Z}\left[u, u^{-1}\right]\right) .
$$

We now group the infinite sum into powers of $u$ having the same value modulo $m$. Let $M$ be a complete set of representatives of the integers modulo $m$, for instance $\{0,1, \ldots, m-1\}$. Then we have

$$
\begin{aligned}
\langle a, b\rangle_{u} & =\frac{\sum_{k \in M} u^{k} \sum_{i=-\infty}^{\infty} I\left(\alpha, u^{i m+k} \beta\right) u^{i m}}{\pi\left(u^{m}\right)} \\
& =\sum_{k \in M} u^{k}\left[\frac{\sum_{i=-\infty}^{\infty} I\left(\alpha, t^{i}\left(u^{k} \beta\right)\right)\left(u^{m}\right)^{i}}{\pi\left(u^{m}\right)}\right] \\
& =\sum_{k \in M} u^{k} \cdot \mu\left\langle a, u^{k} b\right\rangle_{t}
\end{aligned}
$$

where

$$
\mu: \frac{\mathbf{Q}(t)}{\mathbf{Z}\left[t, t^{-1}\right]} \rightarrow \frac{\mathbf{Q}(u)}{\mathbf{Q}\left[u, u^{-1}\right]}
$$

is $f(t) \mapsto f\left(u^{m}\right)$.

2. Given that $\left(A_{t},\langle,\rangle_{t}\right)$ is the module and pairing of such a simple knot, we know it must satisfy the Levine axioms:

(L1) $A_{t}$ is a finitely generated $\Lambda$-torsion-module.

(L2) Multiplication by $(1-t)$ acts as an automorphism of $A_{t}$.

(L3) $\langle,\rangle_{t}$ is $e=(-1)^{q+1}$-Hermitian, that is

$$
\langle a, b\rangle_{t}=e \overline{\langle b, a\rangle_{t}} \text { and } t\langle a, b\rangle_{t}=\langle t a, b\rangle_{t}
$$

where ${ }^{-}$denotes the involution of $\mathbf{Q}(t) / \mathbf{Z}\left[t, t^{-1}\right]$ defined by $\overline{f(t)}=f\left(t^{-1}\right)$.

(L4) $\langle,\rangle_{t}$ is nonsingular, that is, the adjoint map:

$$
A_{t} \rightarrow \operatorname{Hom}_{\Lambda}\left(A_{t}, \mathbf{Q}(t) / \mathbf{Z}\left[t, t^{-1}\right]\right), \quad a \mapsto\left(x \mapsto\langle x, a\rangle_{t}\right)
$$

is a (conjugate-linear) isomorphism.

Furthermore $[\mathbf{K}, \mathbf{T}]$, these conditions characterise the modules and pairings which can arise, together with the condition that the signature of the corresponding quadratic form must be divisible by 16 for $q=2$; that is, any such module and pairing arises as that of a simple knot; and if two knots have isometric pairings, they are equivalent.

To derive the algebraic condition for $k$ to be the $m$-fold branched cyclic cover of a knot, we shall need to use the following trick repeatedly.

LemMA. Suppose $M$ is a set of integers having distinct values modulo $m$ and we are given that

$$
\frac{\sum_{k \in M} \sum_{i=-\infty}^{\infty} a_{k+i m} u^{k+i m}}{\pi\left(u^{m}\right)}=0 \text { in } \frac{\mathbf{Q}(u)}{\mathbf{Z}\left[u, u^{-1}\right]}
$$


Then, for all $k \in M$,

$$
\frac{\sum_{i=-\infty}^{\infty} a_{k+i m} t^{i}}{\pi(t)}=0 \quad \text { in } \frac{\mathbf{Q}(t)}{\mathbf{Z}\left[t, t^{-1}\right]}
$$

Proof. Since

$$
\pi\left(u^{m}\right)\left|\sum_{i=-\infty}^{\infty} b_{i} u^{i} \Leftrightarrow \pi\left(u^{m}\right)\right| \sum_{i=k(\bmod m)} b_{i} u^{i} \quad \forall k \in \mathbf{Z} .
$$

If the module $A_{u}$ and the pairing $\langle,\rangle_{u}$ defined above satisfy the Levine conditions, we may construct a knot $l$ corresponding to them. Let $k^{\prime}$ be the $m$-fold branched cyclic cover of $l$, which is a sphere because $1-u^{m}$ is an automorphism of $A_{u}$. The Alexander module of $k^{\prime}$ is clearly $A_{t}$, and we have

$$
\sum_{k=0}^{m-1} u^{k} \mu\left\langle a, u^{k} b\right\rangle_{t}^{\prime}=\langle a, b\rangle_{u}=\sum_{k=0}^{m-1} u^{k} \mu\left\langle a, u^{k} b\right\rangle_{t}
$$

where $\langle,\rangle_{t}^{\prime}$ is the Blanchfield pairing of $k^{\prime}$. Using the lemma for $k=0(\bmod m)$ we see that $\langle a, b\rangle_{t}=\langle a, b\rangle_{t}^{\prime}$; so $k$ and $k^{\prime}$ are equivalent, and $k$ does arise as an $m$-fold branched cyclic cover. Then we have

THEOREM 1. $\left(A_{u},\langle,\rangle_{u}\right)$ satisfies the Levine conditions (and hence $k$ is an $m$-fold branched cyclic cover $)$ if and only if $u$ is an isometry of $\left(A_{t},\langle,\rangle_{t}\right)$ with $u^{m}=t$.

Proof. First the "only if" part: For $\langle,\rangle_{u}$ to satisfy (L3) we must have

$$
\begin{aligned}
\langle a, b\rangle_{u} & =e \overline{\langle b, a\rangle_{u}} \quad \forall a, b \in A_{u} \\
& \Leftrightarrow \sum_{k=0}^{m-1} u^{k} \mu\left\langle a, u^{k} b\right\rangle_{t}=e \sum_{k=0}^{m-1} \overline{u^{k}} \mu \overline{\left\langle b, u^{k} a\right\rangle_{t}} \\
& =\sum_{k=0}^{m-1} u^{-k} \mu\left\langle u^{k} a, b\right\rangle_{t} \quad \text { as }\langle,\rangle_{t} \quad \text { is } e \text {-Hermitian } \\
& \Leftrightarrow\left\langle a, u^{k} b\right\rangle_{t}=t^{-1}\left\langle u^{m-k} a, b\right\rangle_{t} \quad \forall 0 \leqslant k<m, \text { by the lemma } \\
& \Leftrightarrow\left\langle a, u^{k} b\right\rangle_{t}=\left\langle u^{-k} a, b\right\rangle_{t} \quad \forall 0 \leqslant k<m \\
& \Leftrightarrow u \text { is an isometry of }\langle,\rangle_{t} .
\end{aligned}
$$

Conversely, $A_{u}$ is clearly finitely generated $(\mathrm{Ll})$, and $(1-u)$ is an automorphism since

$$
(1-u)\left(1+u+\cdots+u^{m-1}\right)=1-u^{m}=1-t
$$


So we must prove (L3) and (L4) under the assumption that $u$ is an isometry. We have already proved the first half of (L3) above; for the second half we have

$$
\begin{aligned}
\langle u a, b\rangle_{u} & =\sum_{k=0}^{m-1} u^{k} \mu\left\langle u a, u^{k} b\right\rangle_{t} \\
& =\sum_{k=0}^{m-1} u^{k} \mu\left\langle a, u^{k-1} b\right\rangle_{t} \text { as } u \text { is an isometry } \\
& =u \sum_{k=-1}^{m-2} u^{k} \mu\left\langle a, u^{k} b\right\rangle_{t}=u\langle a, b\rangle_{u} .
\end{aligned}
$$

Finally we prove (L4) in two parts.

(i) The adjoint map is injective. Suppose $\langle a, b\rangle_{u}=0 \forall a \in A_{u}$. Then

$$
\sum_{k=0}^{m-1} u^{k} \cdot \mu\left\langle a, u^{k} b\right\rangle_{t}=0 \quad \forall a \in A_{t} .
$$

We may use the lemma for $k=0$; whence

$$
\langle a, b\rangle_{t}=0 \quad \forall a \in A_{t}
$$

$\therefore b=0$ as required, since $\langle,\rangle_{t}$ is nonsingular.

(ii) The adjoint map is surjective. Suppose $a \mapsto f_{a}$ is a $u$-linear map

$$
A_{u} \rightarrow \mathbf{Q}(u) / \mathbf{Z}\left[u, u^{-1}\right] \text {. }
$$

If $\Delta(t)$ is the Alexander polynomial of $k$, then we may write

$$
f_{a}=\frac{\sum_{k=0}^{m-1} u^{k} f_{a}^{(k)}\left(u^{m}\right)}{\Delta\left(u^{m}\right)} .
$$

Then $a \mapsto f_{a}^{(0)}(t) / \Delta(t)$ is a $t$-linear map

$$
A_{t} \rightarrow \mathbf{Q}(t) / \mathbf{Z}\left[t, t^{-1}\right]
$$

So, as $\langle,\rangle_{t}$ is nonsingular, there is an $x$ in $A_{t}$ such that

$$
\langle a, x\rangle_{t}=f_{a}^{(0)}(t) / \Delta(t) .
$$

Then we have

$$
\begin{aligned}
\langle a, x\rangle_{u} & =\sum_{k=0}^{m-1} u^{k} \mu\left\langle a, u^{k} x\right\rangle_{t} \\
& =\sum_{k=0}^{m-1} u^{k} \mu\left\langle u^{-k} a, x\right\rangle_{t} \text { as } u \text { is an isometry } \\
& =\left(\sum_{k=0}^{m-1} u^{k} f_{u^{-k}}^{(0)}\left(u^{m}\right)\right) / \Delta\left(u^{m}\right) \\
& =\left(\sum_{k=0}^{m-1} u^{k} f_{a}^{(k)}\left(u^{m}\right)\right) / \Delta\left(u^{m}\right) \text { as } a \mapsto f_{a} \text { is } u \text {-linear } \\
& =f_{a} \text { as required. }
\end{aligned}
$$


We have proved that $\langle,\rangle_{u}$ is a genuine Blanchfield pairing, and as we have seen this suffices to demonstrate the theorem for $n>2$. Suppose now that $\langle,\rangle_{t}$ is the Blanchfield pairing of a 3-knot. As the Levine conditions depend only on the value of $q$ modulo two the above proof shows that $\langle,\rangle_{u}$ is the Blanchfield pairing of a 7-knot $l_{7}$ whose $m$-fold cover $k_{7}$ has pairing $\langle,\rangle_{t}$. Any lift of a Seifert surface of $l_{7}$ to the complement of $k_{7}$ will then be a Seifert surface for $k_{7}$; thus the quadratic pairings corresponding to $\langle,\rangle_{t}$ and $\langle,\rangle_{u}$ have the same signature, namely that of the surface; so it follows that if $\langle,\rangle_{t}$ is the Blanchfield pairing of a $3-k n o t$, so is $\langle,\rangle_{u}$, completing the proof.

Finally, we prove a condition for two odd-dimensional simple knots having $k$ as an $m$-fold branched cyclic cover to be equivalent.

THEOREM 2. Suppose $u, v$ are two isometries of $\left(A_{t},\langle,\rangle_{t}\right)$ with $u^{m}=t=v^{m}$. Then $\left(A_{u},\langle,\rangle_{u}\right)$ and $\left(A_{v},\langle,\rangle_{v}\right)$ are isometric, and hence correspond to equivalent knots, if and only if $u$ and $v$ are conjugate by an isometry of $\left(A_{t},\langle,\rangle_{t}\right)$.

Proof. We write both $\langle,\rangle_{u}$ and $\langle,\rangle_{v}$ with values in $\mathbf{Q}(u) / \mathbf{Z}\left[u, u^{-1}\right]$. Then $\phi:\left(A_{u},\langle,\rangle_{u}\right) \rightarrow\left(A_{v},\langle,\rangle_{v}\right)$ is an isometry

$$
\begin{aligned}
& \Leftrightarrow\langle a, b\rangle_{u}=\langle\phi a, \phi b\rangle_{v} \quad \forall a, b \in A_{u} \\
& \Leftrightarrow \sum_{k=0}^{m-1} u^{k} \mu\left\langle a, u^{k} b\right\rangle_{t}=\sum_{k=0}^{m-1} u^{k} \mu\left\langle\phi a, v^{k} \phi b\right\rangle_{t} \quad \forall a, b \in A_{u} \\
& \Leftrightarrow\left\langle a, u^{k} b\right\rangle_{t}=\left\langle\phi a, v^{k} \phi b\right\rangle_{t} \quad \forall a, b \in A_{u}, 0 \leqslant k<m \text { by the lemma } \\
& \Rightarrow \phi \text { is an isometry of }\langle,\rangle_{t} \quad(k=0),
\end{aligned}
$$

and taking $k=1$ we see that $u=\phi^{-1} v \phi$, since $\langle,\rangle_{t}$ is nonsingular.

\section{REFERENCES}

[B] R. C. Blanchfield, Intersection theon of manifolds with operators with applications to knot theory, Ann. of Math. (2) 65 (1957), 340-356.

[K] C. Kearton, Blanchfield duality and simple knots, Trans. Amer. Math. Soc. 202 (1975), 141-160.

[T] H. F. Trotter, On S-equivalence of Seifert matrices, Invent. Math. 20 (1973), 173-207.

Department of Mathematical Sciences, University of Durham, Science Laboratories, South Road, Durham DHI 3LE, England 